\title{
Mapping Conformational Changes in a Self-Assembled Two-Dimensional Molecular Network by Statistical Analysis of Conductance Images
}

\author{
Borislav Naydenov, ${ }^{1,}{ }^{*}$ Samuel Torsney, ${ }^{1}$ Alejandro Santana Bonilla, ${ }^{2}$ Andrea Gualandi, ${ }^{3}$ \\ Luca Mengozzi, ${ }^{3}$ Pier Giorgio Cozzi, ${ }^{3}$ Rafael Gutierrez, ${ }^{2}$ Gianaurelio Cuniberti, ${ }^{2,4,5}$ and \\ John J. Boland ${ }^{1}$ \\ ${ }^{1}$ Centre for Research on Adaptive Nanostructures and Nanodevices (CRANN) \& School of Chemistry, Trinity \\ College Dublin, Dublin 2, Ireland \\ ${ }^{2}$ Institute for Materials Sciences and Max Bergmann Center of Biomaterials, TU Dresden, 01062 Dresden, \\ Germany \\ ${ }^{3}$ ALMA MATER STUDIORUM UNIVERSITÀ DI BOLOGNA, Dipartimento di Chimica "G. Ciamician", Via Selmi \\ 2, 40126 Bologna, Italy \\ ${ }^{4}$ Dresden Center for Computational Materials Science (DCCMS), TU Dresden, 01062 Dresden, Germany \\ ${ }^{5}$ Center for Advancing Electronics Dresden, TU Dresden, 01062 Dresden, Germany
}

(Received 31 August 2018; revised manuscript received 22 January 2019; published 28 March 2019)

A self-assembled two-dimensional (2D) film of tetra-phenyl-porphyrin-4-ferrocene molecules on $\mathrm{Au}(111)$ is studied by STM for the presence of intra- and intermolecular correlations in the configurations of the four-pendant ferrocenyl moieties. A statistical analysis of STS images exploits the Pearson's linear correlation coefficient derived from changes in the molecular electron density across lateral positions in the molecular network as a measure of the intra- and intermolecular coupling and/or conjugation between adjacent equivalent molecular components. Density functional theory (DFT) calculation shows that these electron density changes can be assigned to conformational changes of the ferrocenyl units of the molecules. The methodology presented here can be extended to measure correlations in other 2D systems.

DOI: 10.1103/PhysRevApplied.11.034070

\section{INTRODUCTION}

Molecular-based nanoscale devices [1] have been intensively researched and developed over the last 20 years as alternatives [2-5] to traditional inorganic semiconductorbased electronics. Device assembly necessarily involves the deposition and patterning of ordered molecular layers. As MBE is typically limited to inorganic or lowmolecular-weight systems, the deposition of complex functional molecules for device fabrication purposes frequently involves self-assembly from solution. The need to characterize these deposited molecular layers has led to the development of alternative approaches such as solid-liquid interface scanning tunneling microscopy $[6,7]$. Although this approach provides excellent resolution under ambient conditions, the presence of a solvent limits the accessible bias range and the ability to accurately characterize the electronic properties of the assembled layer. Ultrahigh vacuum (UHV) STM studies provide superior characterization capabilities [8-13]. Among the many applications, STM topographic images have been

\footnotetext{
*naydenob@tcd.ie
}

used for conformer statistics [14] and visualization [15] in disordered as well as in ordered [16] overlayers. Another well-established statistical approach is based on random telegraph noise (RTN) analysis use with which both ordered [17] and disordered [18] films have been studied. Thus, application of STM and scanning tunneling spectroscopy (STS) could lead to greater insights into the overall structure and electronic properties of single-layer molecular networks. In many instances, supporting theoretical modeling is required to provide a more complete understanding. However, in assemblies comprised of functional molecules, the conformational freedom and potential for intra- and intermolecular interactions make modeling intractable and other approaches are required to describe the dynamical interactions within these self-assembled networks.

Here, we report on changes in the LDOS driven by moderate nonperiodic conformational changes in a periodic self-assembled two-dimensional (2D) molecular network. We demonstrate that by statistical analysis of differential conductance images recorded at a specific energy one can quickly estimate the levels of intra- and intermolecular coupling between ferrocenyl moieties of the supramolecular film. 


\section{METHODS AND CHARACTERIZATION}

The system we study here is a single-layer molecular film on a metallic (Au) surface [see Fig. 1(a)] composed of tetra-phenyl-porphyrin (TPP) molecules functionalized in their meso positions with amide substituents comprising ferrocenes (see molecular structure in center of Fig. 1). The film is prepared [19] at room temperature in vacuum $\left(1 \times 10^{-6}\right.$ mbar $)$ on a $\mathrm{Au}(111)$ substrate using commercial electrospray deposition (ESD) from a solution (1:1, Trifluorotoluene : Methanol) that results in the formation of a self-assembled network with a square unit cell of $2.2 \times 2.2 \mathrm{~nm}^{2}$ [19], as shown schematically in Fig. 1(b). The unit cell comprises one whole molecule [see the blue square in Fig. 1(b)] or four ferrocenyl moieties from four adjacent molecules, which we will subsequently refer to as a ferrocenyls quartet [see the green square in Fig. 1(b)].

All tunneling spectroscopy data (spectra and images) presented here are performed at constant current ( $25 \mathrm{pA})$ mode (feedback closed) with lock-in sample-bias modulation of $960 \mathrm{~Hz}(10 \mathrm{mV} \mathrm{rms})$, which is below the current amplifier's (Femto DLPCA-200) upper cut-off frequency.

No spectra normalization is applied due to the flat background in the bias ranges of interest.

This molecular assembly is modeled (including the substrate) by density functional theory (DFT) calculations and was reported previously $[19,20]$. Calculation details with the corresponding references [21-34] are provided in the Supplemental Material [35]. The molecular film formation is dominated by strong $\mathrm{N}-\mathrm{H}-\mathrm{O}$ hydrogen bonds between secondary amide groups of adjacent molecules. The calculated HOMO-LUMO gap of approximately $2.7 \mathrm{eV}$ in the partial electron density of states (PDOS) shown in Fig. 1(d) compares well with the experimental gap of approximately $2.3 \mathrm{eV}$ measured by lowtemperature scanning tunneling spectroscopy at $77 \mathrm{~K}$ as shown in LDOS in Fig. 1(c). The filled-states' peaks $P_{1}$ and $P_{2}$ between -2 and $-1 \mathrm{~V}$ in the LDOS spectrum in

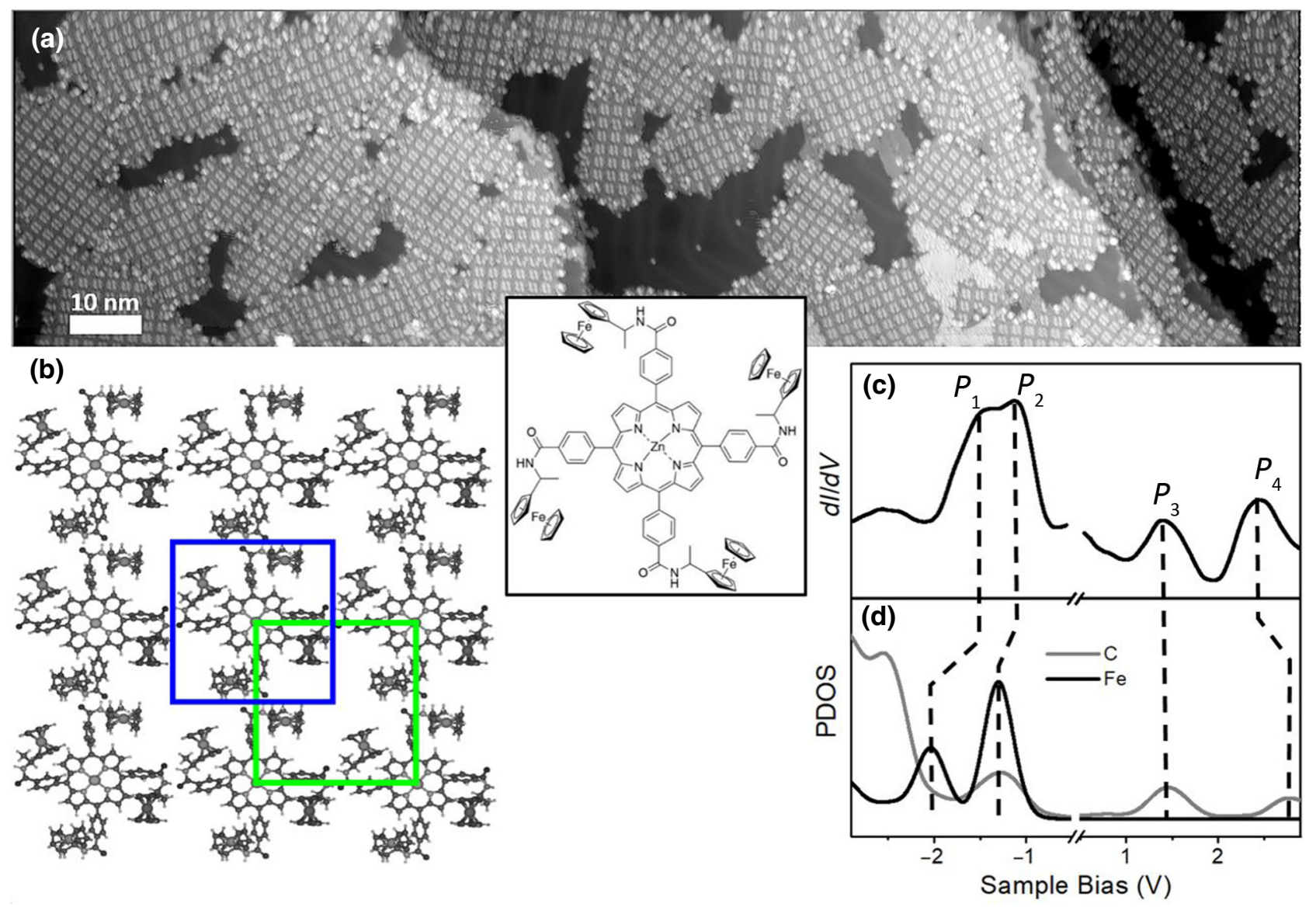

FIG. 1. The chemical structure of the molecule is presented in the center of the figure. (a) Large-area topographic image of a typical 2D molecular network. Tunneling current $15 \mathrm{pA}$, sample bias $+1.0 \mathrm{~V}$. (b) The molecular network model where the square unit cell $\left(2.2 \times 2.2 \mathrm{~nm}^{2}\right)$ is presented comprising one molecule (blue square) or a quartet of adjacent ferrocenyls (green square). (c) Average of differential conductance $(d I / d V)$ spectra measured over different parts of the molecule. (d) Calculated PDOS localized on the carbon atoms (gray line) and on the iron atoms (black line) of the entire molecule. The corresponding peaks in (c) and (d) are marked with dashed lines. 
Fig. 1(c) are dominated by orbitals localized on the iron atoms of the ferrocenyl units, consistent with the PDOS spectra in Fig. 1(d).

\section{RESULTS, STATISTICAL ANALYSIS, AND DISCUSSION}

The STM topographic image presented in Fig. 2(a), which is recorded at $-1.5 \mathrm{~V}$, shows protrusions at the ferrocenyl locations reflecting the integration of tunneling current over the Fe-LDOS doublet $\left(P_{1}-P_{2}\right)$ in Fig. 1(c). The topography in Fig. 2(c) recorded at $+1.5 \mathrm{~V}$ where the integral is over the carbon peak $\left(P_{3}\right)$ in Fig. 1(c) shows intensity at the molecular core and the ferrocenyl locations. The differential-conductance $(d I / d V)$ images, on other hand, show the LDOS intensity at a specific energy in contrast to the integral over an energy range in the topographic images. Thus one should expect a periodic intensity pattern in the conductance images consistent with the network periodicity. This is the case for the image in Fig. 2(d), but not for the image in Fig. 2(b). The latter [Fig. 2(b)] reveals different brightness levels across the network suggesting significant amplitude or positional changes of the high-bias peak $P_{1}$ of the LDOS doublet $\left(P_{1}-P_{2}\right)$ at $-1.5 \mathrm{~V}$ in Fig. $1(\mathrm{c})$.

Since no obvious periodic pattern can be extracted from the conductance image in Fig. 2(b), a statistical analysis of the pixel amplitudes in this conductance image is performed and the resulting sampling distribution is shown in Fig. 2(f). The distribution in Fig. 2(f) shows evidence of three peaks, but to evaluate the reliability of this observation, we perform the exact same analysis of the empty states' LDOS peak $P_{\mathbf{3}}$ at $+1.5 \mathrm{~V}$ in Fig. 1(c). This peak is dominated by the PDOS on the carbon atoms of the molecule as can be seen from Fig. 1(d). The brightness level within the corresponding STM topographic image in Fig. 2(c) is distributed homogenously across the entire molecule while the differential conductance image in Fig. 2(d) shows a strong localization of the $P_{\mathbf{3}}$ peak on the carbon TPP core of the molecule. The pixel-amplitude sampling distribution of the LDOS image in Fig. 2(d) is shown in Fig. 2(h) and contains a single intensity structure, which is well described as a Gaussian with a width of 0.5 arbitrary units. This distribution width comprises the system and experimental broadening of a single peak and enables us to decompose the distribution in Fig. 2(f) into three Gaussian peaks (constant width of 0.6 arbitrary units) corresponding to bright $(I)$, gray (II), and dark (III) intensity levels in Fig. 2(b).

For completeness, the pixel-amplitude distributions of the STM topographic images in Figs. 2(a) and 2(c) are presented in Figs. 2(e) and 2(g), respectively. Not surprisingly, the tunneling current integral over the single peak $P_{3}$ in the LDOS [see Fig. 1(c)] leads to a sharp single feature in the corresponding distribution in Fig. 2(g). On other hand,

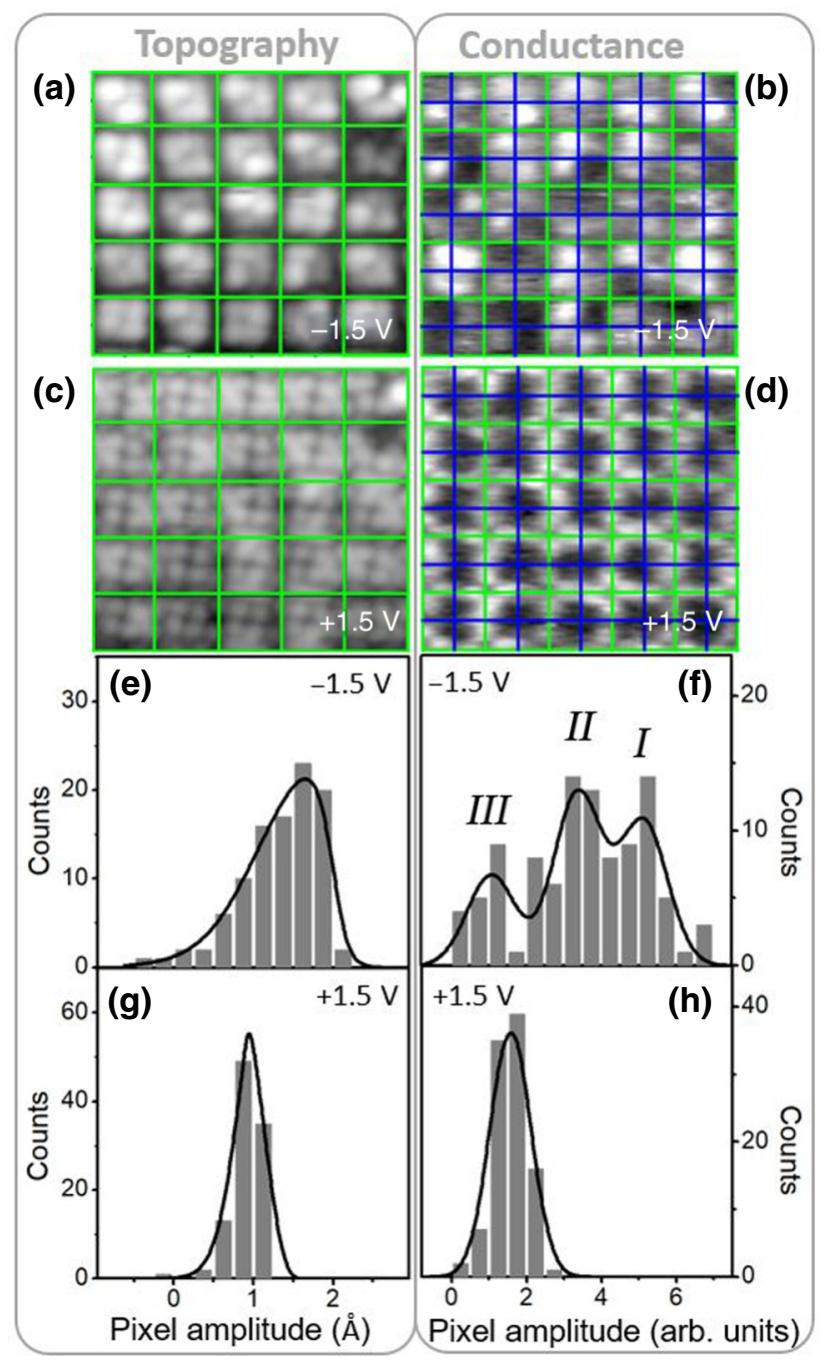

FIG. 2. (a) and (c) Topographic images of the molecular network taken with a tunneling current of $25 \mathrm{pA}$, and sample biases of $-1.5 \mathrm{~V}$ (filled states) and $+1.5 \mathrm{~V}$ (empty states), respectively. The grid represents the network unit cell and each unit is comprised of a quartet of four ferrocenyl units from adjacent molecules [see Fig. 1(b)]. (b) and (d) Differential conductance images of the molecular network taken with a tunneling current of $25 \mathrm{pA}$, and sample biases of $-1.5 \mathrm{~V}$ and $+1.5 \mathrm{~V}$, respectively. Each subgrid unit (pixel) contains one ferrocenyl moiety where blue lines separate the intraquartet neighboring pixels (ferrocenyls) and green lines separate the intramolecule pixels. All images (a)-(d) represent the same area and are recorded by line-by-line alternation of the two biases with a simultaneously recorded tunneling current and its bias derivative. (e)-(h) Pixelamplitude sampling distributions of images (a)-(d), respectively. The pixel area is presented by the grid cell in (b) and (d).

for the filled states' topography in Fig. 2(a), the tunneling integral includes the amplitude and energy shifting of the doublet $\left(P_{1}-P_{2}\right)$ and leads to a broad strongly asymmetric single structure in the distribution in Fig. 2(e), which lacks the three-peak structure resolved in Fig. 2(f). This 
shows the richness available from an analysis of the LDOS changes that is not available from the energy averaged topographic data.

In order to further characterize the nonperiodic pattern in Fig. 2(b), the LDOS spectra in Fig. 3(a) are recorded across three adjacent network unit cells [topographic image shown on the left panel in Fig. 3(a)]. Significant variation of the doublet amplitude and energy position can be observed between the spectra in Fig. 3(a). The spectra cannot be fitted using amplitude variations of peaks with fixed energy positions in the doublet. Significant lateral shifts of the doublet must be included instead. The deviations from the double structure in the experimental data in Fig. 2(b) may be accounted for by spatial LDOS overlap of adjacent ferrocenyls. Additionally, the observed discrepancy between theory and experiment can be understood in terms of the strength of the interface dipole (ID). In general terms, the $I D$ is an effect generated by the interaction of the molecule with a metallic substrate generating a dipole moment that results not only in the reduction of the metallic work function value, but also produces an internal reorganization of the molecular energy levels. In our case, an effective reduction of the $\mathrm{Au}(111)$ work function and molecular energy gaps are computed leading to a lowering of the metallic work function and the ferrocene-associated states of $0.5 \mathrm{eV}$ [19]. Since the experiments are carried out at $T=77 \mathrm{~K}$, the height of the moieties is expected to change as a result of thermal motions producing a change in the intensity of the $I D$, which, in turn, will lead to shifts in the positions of the $P_{1}$ and $P_{2}$ peaks.

To assist in the assignment of the three-peaked pixelamplitude sampling distribution in Fig. 2(f), we turn to the DFT modeling [19] of the molecular network where conformational change of the ferrocenyls is observed [see model in Fig. 3(b)]. The four adjacent ferrocenyls in the quartet adopt different conformers that give rise to slightly shifted peak positions in the corresponding PDOS on the iron atoms of the ferrocenyl moieties [see graphs in Fig. 3(b)]. The filled state doublet feature $\left(P_{1}-P_{2}\right)$ dominated by the iron PDOS in Fig. 3(b) is seen to shift in a manner similar to that observed experimentally in Fig. 3(a), except that theory shows a smaller shift range $(80 \mathrm{mV})$ compared to the experiment $(330 \mathrm{mV})$. Despite this, the parallel shifting of the two peaks is qualitatively similar (details in the Supplemental Material [35]). If we use the distance between the ferrocenyl iron atom and the surface determined from theory as a proxy measure for the different conformers and plot the calculated PDOS peak energy positions of the doublet against it in Fig. 3(c), we observe an exponential behavior of the peak energy position with the $\mathrm{Fe}$ atom above the $\mathrm{Au}$ substrate (Fe height). The same trend is found for each of the doublet peaks $P_{1}$ and $P_{2}$ in Fig. 3(c), consistent with the parallel shifting of the two peaks. The red shift of the peak
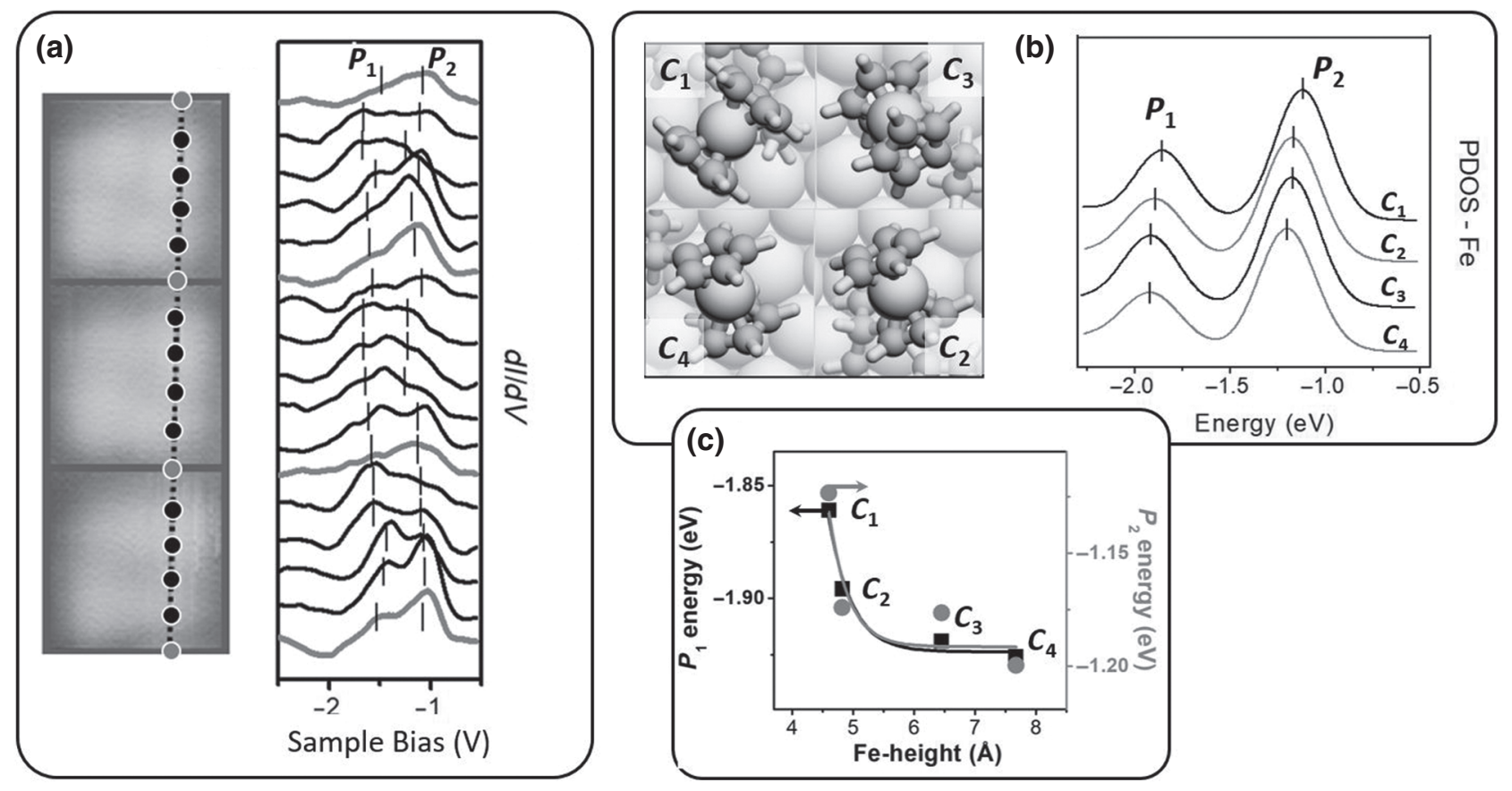

FIG. 3. (a) Differential conductance $(d I / d V)$ spectra taken along the dotted line on a random topographic image on the left-hand side in (a) representing typical spectral behavior in the entire area presented in Fig. 2(a). (b) PDOS localized on the iron atoms of the ferrocenyls in the four conformers (marked $C_{\mathbf{1}}-C_{\mathbf{4}}$ ) and presented in the model on the left-hand side in (b). All spectra in (a) and (b) are vertically translated for better comparison and the peak maxima are marked by vertical bars. (c) Graph of the peak energies of the PDOS-Fe doublet dependence on the Fe height above the gold surface for the different conformers $C_{1}-C_{4}$. 
positions toward lower binding energies with the decrease of the ferrocenyl-surface separation is most likely due to the increasing influence of the molecule-induced image potential in the metal substrate [36]. This image potential effect is generally also involved in the reduction of the HOMO-LUMO gap upon molecular adsorption on surfaces $[37,38]$. The two conformers $\left(C_{3}\right.$ and $\left.C_{4}\right)$, which are furthest away from the surface, have doublet energy positions that are very similar and thus the four conformers are reduced to only three energy resolvable groups, which is consistent with the bright-gray-dark (I-II-III) intensity distribution in Fig. 2(f).

To identify which conformers are associated with each of the three peaks in Fig. 2(f), it is important to remember that the conductance images are recorded at a fixed bias energy of $-1.5 \mathrm{~V}$ and that $P_{1}-P_{2}$ doublet peaks associated with the conformers shift within the tunneling energy window defined by this bias. Since the applied bias is centered on the higher binding energy $P_{1}$ peak, this conformer must correspond to the brightest pixels in Fig. 2(b) and consequently to the bright feature $(I)$ in the distribution in Fig. 2(f). Any shifting of peak positions from the applied bias energy will necessarily result in a reduction of the LDOS amplitude. However, given the asymmetric shape of the experimental $P_{1}-P_{2}$ doublet [Fig. 1(c)], a red shift of the doublet will introduce a larger reduction in the LDOS amplitude compared to a blue shift (see Supplemental Material [35]). Based on these considerations and guided by the PDOS calculations in Fig. 3(b), we assign conformer $C_{2}$ to the $P_{1}$ peak centered at $-1.5 \mathrm{~V}$, which then corresponds to the bright feature $(I)$ in the distribution in Fig. 2(f). The red-shifted $C_{1}$ conformer [see Fig. 3(b)] then corresponds to the dark feature (III) in the distribution in Fig. 2(f), whereas the higher intensity gray feature $(I I)$ is associated with conformers $C_{3}$ and $C_{4}$, which have similar energies. In the absence of coupling between the ferrocenyl conformers, the intensities of features $I$ and $I I I$ in the distribution in Fig. 2(f) should be equal and half that of feature II. Since this is clearly not the case, we now investigate the presence of coupling between the different ferrocenyl conformers.

To this end, we divide the potential ferrocenyl coupling into two types, which we expect to differ, namely, intraquartet (intermolecule) and intramolecule [see green and blue squares in Fig. 1(b)]. Using the differential conductance image from Fig. 2(b), we form pairs of pixel amplitudes for each of the two types of coupling. Each type is decomposed into two sets corresponding to the first and the second nearest neighbors (for details, see Supplemental Material [35]). We statistically analyze the four sets of pixel-amplitude pairs by calculating the Pearson linear correlation coefficient $(r)$ [39]. The diagrams of the four sets are presented in Figs. 4(a)-4(d) with the corresponding correlation coefficient and relative and standard error values. For the ferrocenyls in the quartets, we obtain

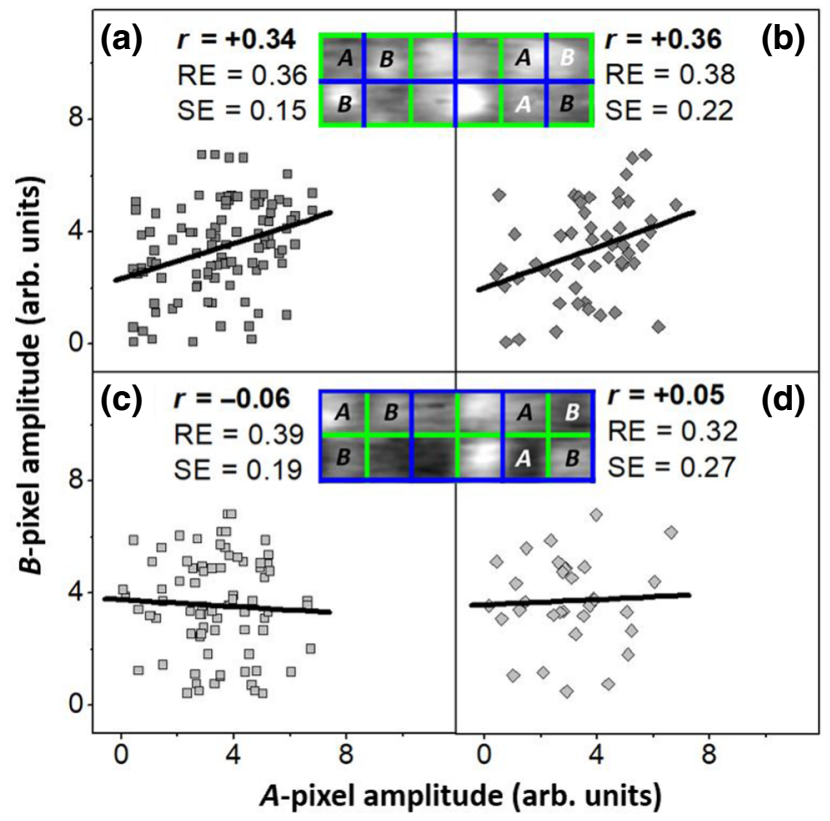

FIG. 4. (a) and (b) Correlation plots of $d I / d V$ pixel amplitudes for first and second nearest neighbor intraquartet pairs $(A-B)$, respectively. (c) and (d) Correlation plots of $d I / d V$ pixel amplitudes for first and second nearest neighbor intramolecular pairs $(A-B)$, respectively. Exemplary pairs $(A-B)$ of the statistical sampling sets are presented in the inserts [from Fig. 2(b)] in the corresponding panels (a)-(d). The values of the correlation coefficient $(r)$, relative (RE), and standard (SE) errors are also presented in panels (a)-(d).

similar correlation coefficients for the first (0.34) and second (0.36) nearest neighbors, consistent with the weak coupling that drives the formation of the ordered layers of ferrocenes on $\mathrm{Cu}(111)$ [40]. The intramolecular correlation coefficient, on the other hand, is very close to zero with values of -0.06 and 0.05 for the first and second nearest neighbors, respectively, indicating that there is no coupling between the ferrocenyls across the molecule. The latter is expected as no conjugation between the phenyl and the porphyrin is observed in the calculations. The weak level of interaction between the ferrocenyl side groups is also consistent with an electrochemical study [20] of a very similar supramolecular film where a single oxidation peak is observed by cyclic voltammograms, which corresponds to the presence of four independent and equivalent ferrocenyl units.

\section{CONCLUSION}

Differential conductance images of a tetra-phenylporphyrin-4-ferrocene self-assembled 2D film on $\mathrm{Au}(111)$ reveal the presence of STS peaks associated with the ferrocenyl moieties. The energies of these peaks shift depending on the local conformation, which is consistent with theory. An intensity analysis allows an assignment of conformers 
with those identified by theory and reveals a nonrandom distribution of conformers across the network. A spatial analysis of intensity changes provides an estimate of the level of coupling between the ferrocenyl units within and between molecules. A weak attraction (correlation coefficient: $r=0.35$ ) is observed in the case of intermolecular coupling, which is absent $(r \sim 0)$ in the case of intramolecular ferrocenyl coupling. The statistical analysis of the differential conductance images described here is enabled by the optimal choice of a specific STS bias where molecular electron density changes occur due to the ferrocenyl conformational changes. This characterization approach is expected to be generally useful for the study of nonperiodic changes and phenomena in periodic $2 \mathrm{D}$ systems.

\section{ACKNOWLEDGMENTS}

This work was supported by the European Community through the Grant No. EC FP7 ICT-MOLARNET (318516). This work has also been partly supported by the International Center for Frontier Research in Chemistry (icFRC) and the German Research Foundation (DFG) within the Cluster of Excellence "Center for Advancing Electronics Dresden." Computational resources were provided by the ZIH at Dresden University of Technology.

B.N., S.T., and J.B. were involved in the STM/STS experiments, analysis, discussions, and manuscript preparation. A.S.B., R.G., and G.C. were involved in the theoretical modeling and in the discussions. A.G., L.M., and P.G.C. were involved in the molecular design and synthesis.

[1] J. C. Dong, B. A. Parviz, M. Hong, and A. K. Y. Jen, Using self-assembly for the construction of nanoscale lateraltransport molecular electronic devices and microscale silicon-based networks, Nanosensing: Mater. Devices 5593, 112 (2004).

[2] M. Berggren, D. Nilsson, and N. D. Robinson, Organic materials for printed electronics, Nat. Mater. 6, 3 (2007).

[3] R. M. Metzger, Unimolecular electronics, J. Mater. Chem. 18, 4364 (2008).

[4] S. M. Tong, J. Song, and Q. D. Ling, Polymer electrical memory materials and diode memory devices, Prog. Chem. 23, 1700 (2011).

[5] J. D. Myers and J. G. Xue, Organic semiconductors and their applications in photovoltaic devices, Polym. Rev. 52, 1 (2012).

[6] R. Sonnenfeld and P. K. Hansma, Atomic-resolution microscopy in water, Science 232, 211 (1986).

[7] S. De Feyter and F. C. De Schryver, Two-dimensional supramolecular self-assembly probed by scanning tunneling microscopy, Chem. Soc. Rev. 32, 139 (2003).

[8] H. Spillmann, A. Kiebele, M. Stohr, T. A. Jung, D. Bonifazi, F. Y. Cheng, and F. Diederich, A two-dimensional porphyrin-based porous network featuring communicating cavities for the templated complexation of fullerenes, Adv. Mater. 18, 275 (2006).

[9] W. Auwarter, A. Weber-Bargioni, A. Riemann, A. Schiffrin, O. Groning, R. Fasel, and J. V. Barth, Self-assembly and conformation of tetrapyridyl-porphyrin molecules on $\operatorname{Ag}(111)$, J. Chem. Phys. 124, 194708 (2006).

[10] N. Wintjes, D. Bonifazi, F. Y. Cheng, A. Kiebele, M. Stohr, T. Jung, H. Spillmann, and F. Diederich, A supramolecular multiposition rotary device, Angew. Chem. Int. Ed. 46, 4089 (2007).

[11] B. A. Friesen, B. Wiggins, J. L. McHale, U. Mazur, and K. W. Hipps, Differing HOMO and LUMO Mediated Conduction in a Porphyrin Nanorod, J. Am. Chem. Soc. 132, 8554 (2010).

[12] D. Ecija, K. Seufert, D. Heim, W. Auwarter, C. Aurisicchio, C. Fabbro, D. Bonifazi, and J. V. Barth, Hierarchic self-assembly of nanoporous chiral networks with conformationally flexible porphyrins, ACS Nano 4, 4936 (2010).

[13] T. A. Pham, F. Song, M. N. Alberti, M. T. Nguyen, N. Trapp, C. Thilgen, F. Diederich, and M. Stohr, Heatinduced formation of one-dimensional coordination polymers on $\mathrm{Au}(111)$ : an STM study, Chem. Commun. 51, 14473 (2015)

[14] I. Lee, S. Son, T. Shin, and J. R. Hahn, Direct observation of the conformational transitions of single pyridine molecules on a $\operatorname{Ag}(110)$ surface induced by long-range repulsive intermolecular interactions, J. Chem. Phys. 146, 014706 (2017).

[15] B. N. Taber, D. A. Kislitsyn, C. F. Gervasi, J. M. Mills, A. E. Rosenfield, L. Zhang, S. C. B. Mannsfeld, J. S. Prell, A. L. Briseno, and G. V. Nazin, Real-space visualization of conformation-independent oligothiophene electronic structure, J. Chem. Phys. 144, 194703 (2016).

[16] N. Kalashnyk, L. Amiaud, C. Dablemont, A. Lafosse, K. Bobrov, and L. Guillemot, Strain relaxation and epitaxial relationship of perylene overlayer on $\mathrm{Ag}(110)$, J. Chem. Phys. 148, 214702 (2018).

[17] H. R. Wu, K. Sotthewes, A. Mendez-Ardoy, T. Kudernac, J. Huskens, A. Lenferink, C. Otto, P. M. Schon, G. J. Vancso, and H. J. W. Zandvliet, Dynamics of oligo(phenylene-ethynylene) self-assembled monolayers on $\mathrm{Au}(111)$, Chem. Phys. Lett. 614, 45 (2014).

[18] R. Thamankar, N. Raghavan, J. Molina, F. M. Puglisi, S. J. O'Shea, K. Shubhakar, L. Larcher, P. Pavan, A. Padovani, and K. L. Pey, Single vacancy defect spectroscopy on $\mathrm{HfO}_{2}$ using random telegraph noise signals from scanning tunneling microscopy, J. Appl. Phys. 119, 084304 (2016).

[19] B. Naydenov, S. Torsney, A. S. Bonilla, M. El Garah, A. Ciesielski, A. Gualandi, L. Mengozzi, P. G. Cozzi, R. Gutierrez, P. Samorì, G. Cuniberti, and John J. Boland, Self-assembled 2D supramolecular networks characterized by STM/STS in air and under vacuum, Langmuir 34, 7698 (2018).

[20] M. El Garah, A. S. Bonilla, A. Ciesielski, A. Gualandi, L. Mengozzi, A. Fiorani, M. Iurlo, M. Marcaccio, R. Gutierrez, S. Rapino, M. Calvaresi, F. Zerbetto, G. Cuniberti, P. G. Cozzi, F. Paolucci, and P. Samori, Molecular design driving tetraporphyrin self-assembly on graphite: a joint STM, electrochemical and computational study, Nanoscale 8, 13678 (2016). 
[21] M. Frigo and S. G. Johnson, The design and implementation of FFTW3, Proc. IEEE 93, 216 (2005).

[22] J. Hutter, M. Iannuzzi, F. Schiffmann, and J. VandeVondele, CP2K: atomistic simulations of condensed matter systems, Wiley Interdiscip. Rev. Comput. Mol. Sci. 4, 15 (2014).

[23] M. Krack and M. Parrinello, All-electron ab-initio molecular dynamics, Phys. Chem. Chem. Phys. 2, 2105 (2000).

[24] G. Lippert, J. Hutter, and M. Parrinello, The Gaussian and augmented-plane-wave density functional method for $a b$ initio molecular dynamics simulations, Theor. Chem. Acc. 103, 124 (1999).

[25] J. VandeVondele and J. Hutter, An efficient orbital transformation method for electronic structure calculations, J. Chem. Phys. 118, 4365 (2003).

[26] J. VandeVondele and J. Hutter, Gaussian basis sets for accurate calculations on molecular systems in gas and condensed phases, J. Chem. Phys. 127, 114105 (2007).

[27] M. Krack and M. Parrinelli, QUICKSTEP: Make the atoms dance, high performance, Comput. Chem. 25, 29 (2004).

[28] J. VandeVondele, M. Krack, F. Mohamed, M. Parrinello, T. Chassaing, and J. Hutter, QUICKSTEP: Fast and accurate density functional calculations using a mixed Gaussian and plane waves approach, Comput. Phys. Commun. 167, 103 (2005).

[29] G. Lippert, J. Hutter, and M. Parrinello, A hybrid Gaussian and plane wave density functional scheme, Mol. Phys. 92, 477 (1997).

[30] M. Guidon, J. Hutter, and J. VandeVondele, Auxiliary density matrix methods for Hartree-Fock exchange calculations, J. Chem. Theory Comput. 6, 2348 (2010).
[31] J. Spencer and A. Alavi, Efficient calculation of the exact exchange energy in periodic systems using a truncated Coulomb potential, Phys. Rev. B 77, 193110 (2008).

[32] M. Guidon, J. Hutter, and J. VandeVondele, Robust periodic Hartree-Fock exchange for large-scale simulations using Gaussian basis sets, J. Chem. Theory Comput. 5, 3010 (2009).

[33] C. Adamo and V. Barone, Toward reliable density functional methods without adjustable parameters: The PBE0 model, J. Chem. Phys. 110, 6158 (1999).

[34] J. P. Perdew, K. Burke, and M. Ernzerhof, Generalized Gradient Approximation Made Simple, Phys. Rev. Lett. 77, 3865 (1996).

[35] See Supplemental Material at http://link.aps.org/supple mental/10.1103/PhysRevApplied.11.034070 for additional STS data and statistical analysis.

[36] J. Bardeen, Electron correlation and screening effects - in relation to surface physics, Surf. Sci. 2, 381 (1964).

[37] R. Hesper, L. H. Tjeng, and G. A. Sawatzky, Strongly reduced band gap in a correlated insulator in close proximity to a metal, Europhys. Lett. 40, 177 (1997).

[38] J. B. Neaton, M. S. Hybertsen, and S. G. Louie, Renormalization of Molecular Electronic Levels at Metal-Molecule Interfaces, Phys. Rev. Lett. 97, 216405 (2006).

[39] H. Frank and S.C. Althoen, Statistics: Concepts and Applications (Cambridge University Press, New York, NY, USA, 1994), 113.

[40] B. W. Heinrich, L. Limot, M. V. Rastei, C. Iacovita, J. P. Bucher, D. M. Djimbi, C. Massobrio, and M. Boero, Dispersion and Localization of Electronic States at a Ferrocene/Cu(111) Interface, Phys. Rev. Lett. 107, 216801 (2011). 